\title{
BMJ Open Dietary assessment of British police force employees: a description of diet record coding procedures and cross- sectional evaluation of dietary energy intake reporting (The Airwave Health Monitoring Study)
}

Rachel Gibson, ${ }^{1}$ Rebeca Eriksen, ${ }^{1}$ Kathryn Lamb, ${ }^{1}$ Yvonne McMeel, ${ }^{1}$ Anne-Claire Vergnaud, ${ }^{2}$ Jeanette Spear, ${ }^{2}$ Maria Aresu, ${ }^{2}$ Queenie Chan, ${ }^{2}$ Paul Elliott, ${ }^{2}$ Gary Frost ${ }^{1}$

To cite: Gibson R, Eriksen R, Lamb K, et al. Dietary assessment of British police force employees: a description of diet record coding procedures and cross-sectional evaluation of dietary energy intake reporting (The Airwave Health Monitoring Study). BMJ Open 2017;7:e012927. doi:10.1136/bmjopen-2016012927

- Prepublication history and additional material are available. To view please visit the journal (http://dx.doi.org/ 10.1136/bmjopen-2016012927).

Received 2 June 2016 Revised 2 November 2016 Accepted 23 December 2016

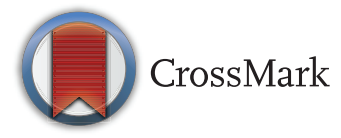

For numbered affiliations see end of article.

Correspondence to Professor Gary Frost; g.frost@imperial.ac.uk

\section{ABSTRACT}

Objectives: Dietary intake is a key aspect of occupational health. To capture the characteristics of dietary behaviour that is affected by occupational environment that may affect disease risk, a collection of prospective multiday dietary records is required. The aims of this paper are to: (1) collect multiday dietary data in the Airwave Health Monitoring Study, (2) describe the dietary coding procedures applied and (3) investigate the plausibility of dietary reporting in this occupational cohort.

Design: A dietary coding protocol for this large-scale study was developed to minimise coding error rate. Participants (n 4412) who completed 7-day food records were included for cross-sectional analyses. Energy intake (EI) misreporting was estimated using the Goldberg method. Multivariate logistic regression models were applied to determine participant characteristics associated with El misreporting.

Setting: British police force employees enrolled (2007-2012) into the Airwave Health Monitoring Study.

Results: The mean code error rate per food diary was $3.7 \%$ (SD 3.2\%). The strongest predictors of El under-reporting were body mass index (BMI) and physical activity. Compared with participants with $B M \mid<25 \mathrm{~kg} / \mathrm{m}^{2}$, those with BMI $>30 \mathrm{~kg} / \mathrm{m}^{2}$ had increased odds of being classified as under-reporting El (men OR $5.2095 \% \mathrm{Cl} 3.92$ to 6.89 ; women OR $2.6695 \% \mathrm{Cl} 1.85$ to 3.83 ). Men and women in the highest physical activity category compared with the lowest were also more likely to be classified as underreporting (men OR 3.33 95\% Cl 2.46 to 4.50; women OR 4.34 95\% Cl 2.91 to 6.55 ).

Conclusions: A reproducible dietary record coding procedure has been developed to minimise coding error in complex 7-day diet diaries. The prevalence of El under-reporting is comparable with existing national UK cohorts and, in agreement with previous studies,

\section{Strengths and limitations of this study}

- The Airwave Health Monitoring Study provides the largest collection and assessment of 7-day food records from a single occupation UK cohort.

- A comprehensive and reproducible diet record coding procedure has been developed to minimise coding error in this study.

- A large number of occupational as well as sociodemographic variable measures facilitate the investigation of a wide range of factors potentially associated with energy intake (EI) misreporting.

- Self-report physical activity and dietary data highlight the common limitations of estimating accuracy of dietary El reporting in large nutritional epidemiological studies

classification of under-reporting was biased towards specific subgroups of participants.

\section{INTRODUCTION}

The Airwave Health Monitoring Study is a longitudinal study of British police force employees launched in 2004. ${ }^{1}$ This study is the largest cohort of police employees worldwide with 42112 participants enrolled into the study at the end of 2012 with a high proportion of men in early adulthood who are unrepresented in existing UK longitudinal studies. ${ }^{2}$ Data from the voluntary healthscreening programme include extensive occupational, medical, biochemical, cognitive and 
lifestyle information. Since April 2007, participants completed a 7-day estimated weight food diary (n 15 404). One of the limitations of previous research in large-scale occupational cohort studies is that retrospective methods of dietary data collection, such as food frequency questionnaires, ${ }^{34}$ rather than prospective methods have been used to investigate dietary behaviour. Therefore, the large-scale collection of 7-day food records from a single occupational group makes the Airwave Health Monitoring Study unique, as it will allow the comprehensive investigation of diet and various occupational factors with health outcomes.

The benefit of prospective measurement methods such as diet diaries compared with food frequency questionnaires is that they allow more detailed dietary intake to be captured, as they do not measure against a predefined food list. Additionally, compared with food records they are less reliant on participant recall. Prospective measures such as diet diaries provide important information about eating occasions, frequency of eating, regularity and the combination of foods consumed. Research increasingly suggests that measuring these additional aspects of dietary behaviour provides a holistic understanding about the relationship between diet and various chronic metabolic diseases. ${ }^{5-8}$ Despite the valuable information generated from diet diaries, it is widely acknowledged that all current dietary measurement tools present a challenge to nutritional scientists as they are subject to human error at each stage of the assessment process. First, there may be either intentional or unintentional misreporting of dietary intake by participants. ${ }^{9}$ For example, prospective dietary reporting may result in conscious or unconscious changes in diet intake during the period of observation. This is of particular concern, as energy balance is an established risk factor in the aetiology of chronic metabolic diseases. It is also acknowledged that dietary misreporting may be associated with specific population groups. ${ }^{10}{ }^{11}$ Therefore, an important part of the methodological process in nutritional epidemiology is to investigate the plausibility of dietary energy reporting and to identify participant characteristics associated with implausible reporting to avoid bias and erroneous conclusions. ${ }^{12}$ The second stage open to error is the 'coding' of food records (the matching of food and drink items recorded to a nutritional database code and a portion size) which is prone to subjective decision-making even by experienced coders. ${ }^{13}$

The recently published Strengthening the Reporting of Observational Studies in Epidemiology-Nutritional Epidemiology (STROBE-nut) statement recommends transparency in the methods used to derive nutritional data and the investigation of potential sources of bias in dietary reporting. ${ }^{14}$ In line with STROBE-nut recommendations, the aims of this paper are (1) to describe the dietary data coding methods applied to the Airwave Health Monitoring Study cohort, (2) to investigate the plausibility of energy reporting among the Airwave
Health Monitoring Study participants and (3) to identify the characteristics associated with energy intake (EI) misreporting. We also conducted exploratory analyses to determine if diet code error rate was associated with implausible EIs. The results of this study will characterise dietary energy reporting within the Airwave Health Monitoring Study and will be used in subsequent studies to guide the statistical treatment of nutritional intakes within this cohort.

\section{METHODS}

The Airwave Health Monitoring Study is conducted according to the guidelines laid down in the Declaration of Helsinki. Written informed consent was obtained from all participants.

\section{Description of dietary record coding methods Dietary measurement}

The Airwave Health Monitoring Study was open to all police forces in Great Britain. Recruitment procedures have been previously described in detail. ${ }^{1}$ Dietary intake was measured using 7-day estimated weight food diaries previously validated against urinary and blood biomarkers in a large-scale UK epidemiological cohort. ${ }^{15}$ The food diary was posted to participants with detailed written instructions to record all food and drink consumed over seven consecutive days in predefined eating occasions. Participants were asked to provide details on cooking methods, brand names and portion sizes. To aid portion size estimation, photographs were provided based on those developed by Nelson and Haraldsdottir. ${ }^{16}$

\section{Dietary data generation}

Calculation of nutritional intake was conducted using Dietplan6.7 software (Forestfield Software, Horsham, UK) which is based on the McCance and Widdowson's 6th Edition Composition of Foods UK Nutritional Data set (UKN). A team of trained coders 'coded' the diaries (matching of food and drink items recorded to a UKN database code and a portion size). Diaries were excluded from coding when $<1$ day was completed or if a meal replacement diet was recorded. A standard operating protocol was developed to reduce the number of subjective decisions made by coders. It provides a series of flow diagrams to guide coders in the translation of food and drink records to database codes and portion sizes to weights (grams) using published recourses of portion $^{17} 18$ and food density information. ${ }^{18} 19$ The standard operating protocol is available as a supplmentary document (see online supplementary document). In conjunction with the standard protocol, a 'codebook' has been developed to assist decision-making when no exact UKN code match can be found. The codebook is an evolving database containing $>600$ online supplementary codes and coding rules following the principles of the codebook designed by Conway et al for 
use in the International collaborative of macronutrients, micronutrients and blood pressure (INTERMAP) study. ${ }^{20}$ Examples of different scenarios and possible coding solutions are shown in online supplementary material table S1.

\section{Coder training}

To date, 20 dietary coders have been trained in the use of Dietplan6.7 software and the Airwave Health Monitoring Study standard protocol. The time an individual coder works on the project varies between 3 months to 2 years. All trainees are required to code ten 'test' food diaries using the standard protocol and codebook before progressing to code diaries from the study. A research dietitian or nutritionist checks the completed electronic Dietplan6.7 record against the written diary for coding errors. Individual feedback is given to the trainee coder after each test diary is completed. At this time, if the total errors are $>10 \%$ per diary, the coder will be required to complete further test diaries until errors are within tolerance.

\section{Quality checking}

An audit cycle has been developed to monitor intercoder reliability with the aim of continuously improving coding consistency. Five per cent of all coded diaries are selected at random every 2 months. Research dietitians and nutritionists check the selected electronic Dietplan6.7 record against the written food diary and classify errors as: 'code selection error' (the code selected in Dietplan6.7 does not match the written record), 'portion error' (over $\pm 10 \%$ difference of the protocol weight), 'meal code error' (item entered into incorrect meal occasion), 'missing code error' (item not coded, ie, in the written record) and 'extra code error' (item coded, ie, not in the written record). If the error rate in an audit check diary is $>10 \%$, feedback and training is provided to the individual coder. Following each audit cycle, the results are fed back to the team and coding improvement strategies are implemented as indicated, for example, staff training, new codebook entries and the development of additional protocol flow diagrams. The final data set is screened for gross coding errors. A gross coding error is defined as when the quantity of food recorded is a clear code error, ${ }^{21}$ for example, entering $260 \mathrm{~g}$ of instant coffee powder rather than $260 \mathrm{~g}$ of instant coffee made with water. If the quantity of any item coded exceeded the set maximum portion, the food diary barcode was identified and the original diary record checked and the quantity amended.

\section{Evaluating reported EI \\ Participants}

Inclusion criteria for the present cross-sectional analysis were men and women enrolled into the Airwave Health Monitoring Study between 2007 and 2012 who had health screen and coded dietary data without gross coding errors ( $n$ 4412). Dietary energy under-reporting methods assume a stable bodyweight, therefore participants were excluded from the analyses if they were pregnant $(n 0)$ and/or reported being on a weight-loss diet at the time of the health screen $(n 317)$. Two participants were excluded due to extremely low EI reporting of $<500 \mathrm{kcal} /$ day, which is considered to be physiologically unsustainable.

\section{Non-dietary variable measurements}

During the health screen, participants were asked to complete a self-administered questionnaire on a touch screen computer to recorded information about occupational, lifestyle, medical history, socioeconomic and demographic factors. Total working hours were taken as the sum of 'regular weekly working hours' plus 'usual overtime hours' and classified into four groups $(<41$, $41-48,49-54$ and $>55$ hours per week) based on previous research. ${ }^{22}$ Standard police employment rank for officers was selected from a predetermined list (constable/sergeant, inspector/chief inspector and superintendent/higher). Participants not employed as police officers were classified as 'staff'. Job descriptions were collapsed from 31 to two categories of 'job role' based on predominant working environment (mobile or office based). Trained nurses took anthropometric measurements following a standard protocol. Bodyweight was measured to the nearest $0.05 \mathrm{~kg}$ using digital scales (Marsden digital weighing scale). Standing height was measured to the nearest $0.1 \mathrm{~cm}$ (Marsden H226 portable stadiometer). Body mass index $\left(\mathrm{BMI} \mathrm{kg} / \mathrm{m}^{2}\right)$ was classified as per WHO cut-offs: underweight $<18.5 \mathrm{~kg} / \mathrm{m}^{2}$; healthy $18.5-24.99 \mathrm{~kg} / \mathrm{m}^{2}$; overweight $25-29.99 \mathrm{~kg} / \mathrm{m}^{2}$ and obese $>30 \mathrm{~kg} / \mathrm{m}^{2} .^{23}$ Physical activity information was collected using The International Physical Activity Questionnaire-Short Form (IPAQ-SF) ${ }^{24}$ which calculates metabolic equivalent (MET) minutes per week across three exercise parameters (walking, moderate and vigorous). The IPAQ-SF protocol was followed to classify each participant as achieving a high, moderate or low level of activity. ${ }^{25}$ As the physical activity data generated by IPAQ-SF do not cover a 24-hour period to permit translation to an overall physical activity level (PAL) value, we assigned estimated PALs of 1.4, 1.6 and 1.8 to 'low', 'moderate' and 'high' IPAQ-SF MET classification, respectively. These values are based on published Department of Health guidance representing nonoccupational and occupational activity levels. ${ }^{26}$ To check the rationale of this assumption, we compared IPAQ-MET classification with self-reported body type. Self-reported body type was asked by the research nurse as part of the bioelectrical impedance measurement protocol (Tanita BC-418MA body composition analyser). 'Athletic' refers to intense exercise ( $>10$ hours intense exercise per week) and 'standard' to $<10$ hours intense exercise per week. The agreement between those selfclassified as 'athletic' and being in the highest IPAQ-SF MET category was $100 \%$. To explore if coder error rate was related to EI misreporting, we classified coders 
dichotomously (mean error rates above/below the overall mean error rate of $3.7 \%$ ).

\section{Classification of El misreporting}

Potential misreporting of EI reporting is based on the assumption that participants are weight stable, where estimated EI is equal to estimated total energy expenditure (TEE). EI was calculated as the mean daily EI recorded across the number of days the food diary was completed. TEE is expressed as estimated basal metabolic rate (BMR) multiplied by an estimated PAL value. We estimated individual BMR (kcal/day) using Schofield equations based on sex, age and weight; ${ }^{26}$ we then assigned PAL values based on the MET category and applied the Goldberg equation. ${ }^{27}$ This equation takes into account the estimated variation in daily EI, BMR and PAL based on previous studies and the number of days of diet assessment. ${ }^{27}$ CIs were calculated for each participant based on PAL values and days of food diary completion. Participants with a ratio of EI: BMR below the lower $95 \%$ CI cut-off value were classified as possibly under-reporting and above the higher cut-off as potentially over-reporting EI. There were 15 $(0.3 \%)$ participants classified as over-reporting EI; these were removed from subsequent analyses due to their small number, providing a final analytical sample size of 4078.

\section{Statistical methods}

Statistical analyses were undertaken using Statistical Analysis System's statistical software V.9.3 (SAS Institute, Cary, North Carolina, USA). All statistical tests are two sided. The sample was stratified by sex to allow estimation of sex-specific associations between variables and the likelihood of implausible energy reporting. To assess differences between two groups, independent t-tests were used for data with normal distribution and Mann-Whitney $\mathrm{U}$ test otherwise. Mean and SD were reported for data with normal distribution and median and IQR otherwise. Associations with categorical variables were analysed using test $\left(\chi^{2}\right)$. Sex-stratified stepwise logistic regression was conducted to identify variables that showed a statistically significant association with likelihood of underreporting EI. Initially, we included all variables known to be previously associated with EI misreporting (age, BMI, smoking status, household income, physical activity, ethnicity, marital status and education) and occupational specific covariates related to the Airwave Health Monitoring Study (police rank, job role, number of working hours, time sitting per weekday). Shift work was not included in the model due to the small sample size with available data $(n 536)$. Job role $(n 2875)$ was not a significant predictor of under-reporting in the initial models, and due to its significant association with rank $\left(\chi^{2}, \mathrm{p}=0.048\right)$ and weekday sitting $\left(\chi^{2}, \mathrm{p}<0.0001\right)$, this variable was not included in the final model to enable the maximum sample size with complete data to be included for analysis. Variables with $\mathrm{p}<0.05$ were included in the final model. Two sets of sensitivity analyses were conducted: one removing participants who reported being on a special diet not for weight loss ( $n$ 171), and one removing those that recorded a change in appetite in previous 2 weeks ( $n 834)$.

\section{RESULTS}

\section{Dietary data quality control}

The mean code error rate detected through the initial 10 audit cycles was $3.7 \%$ (SD 3.2\%) errors per food diary. The mean error range across 10 audit cycles was $2.8 \%$ (SD $3.5 \%$ ) to $5.9 \%$ (SD $4.1 \%$ ) with only one food diary exceeding the $10 \%$ code error limit $(10.2 \%$ error rate). Analysis of audit cycle errors found that the most frequent coding errors were portion weight errors $(55 \%$ of errors detected), followed by code selection errors (31\% of errors detected). Common errors detected during the audit cycles shown in online supplementary material table S2. Gross coding errors were detected in $12 \%$ of food diaries coded.

\section{Population characteristics}

The proportion of food diaries completed for the entire 7-day period was $88 \%$; mean period of diary completion was 6.8 (SD 0.7) days. The characteristics of the sample by sex are shown in table 1 . Men accounted for $63 \%$ of the sample and were significantly older than women: 42.4 (SD 8.9) vs 39.9 (SD 9.5) years, $\mathrm{p}<0.0001$, and had a higher BMI: 27.7 (SD 3.6) $\mathrm{kg} / \mathrm{m}^{2}$ vs 25.6 (SD 4.6) $\mathrm{kg} / \mathrm{m}^{2}$, $\mathrm{p}<0.0001$. Women reported a significantly lower mean daily EI compared with men: 1711 (SD 395) kcal per day versus2107 (SD 502) kcal per day; $\mathrm{p}<0.0001$. There were significant differences between sources of EI between men and women, with women deriving more energy from carbohydrates $(\mathrm{p}<0.0001)$ and men deriving more energy from fats $(p=0.004)$, proteins $(p=0.001)$ and alcohol $(\mathrm{p}<0.0001)$

\section{Classification of under-reporting}

Sex was significantly associated with under-reporting EI with $56 \%$ of men compared with $41 \%$ of women being classified as under-reporting EI $(\mathrm{p}<0.0001)$. The overall prevalence of likely under-reporting of EI was $49 \%$. Sex-stratified analyses showed differences in the associations between demographic, lifestyle and occupational factors when under-reporters and plausible reporters were compared as presented in table 2. Across men and women, potential under-reporters were more likely to be classified as overweight or obese compared with plausible reporters $(\mathrm{p}<0.0001)$, and to be in the highest category for physical activity $(p<0.0001)$. Male police staff were more likely to be classified as plausible reporters, while constable and sergeants were more likely to be classified as under-reporters $(p=0.038)$. Men in the highest quartile for weekday sitting (10 to 13 hours per day) were more likely to be classified as a plausible reporter compared with the lowest quartile ( $<4$ hours 
Table 1 Characteristics of participants with dietary records from the Airwave Health Monitoring Study

\begin{tabular}{|c|c|c|c|c|c|}
\hline & \multicolumn{2}{|c|}{ Men (n 2568) } & \multicolumn{2}{|c|}{ Women (n 1510) } & \multirow[b]{2}{*}{ p Value } \\
\hline & Mean & SD & Mean & SD & \\
\hline Age at screening, years & 42.4 & 8.9 & 39.9 & 9.5 & $<0.0001^{*}$ \\
\hline Body mass index, $\mathrm{kg} / \mathrm{m}^{2}$ & 27.7 & 3.6 & 25.6 & 4.6 & $<0.0001^{*}$ \\
\hline Basal metabolic rate, kcal per day & 1906.0 & 159.0 & 1431.0 & 124.0 & $<0.0001^{\star}$ \\
\hline Daily energy, kcal & 2107.0 & 502.0 & 1711.0 & 395.0 & $<0.0001^{*}$ \\
\hline Energy intake: basal metabolic rate & 1.1 & 0.3 & 1.2 & 0.6 & $<0.0001^{*}$ \\
\hline Macronutrient breakdown of energy intake & Median & IQR & Median & IQR & \\
\hline$\%$ Energy intake carbohydrate & 43.9 & 6.3 & 44.9 & 6.3 & $<0.0001^{\star}$ \\
\hline$\%$ Energy intake protein & 17.0 & 9.5 & 16.7 & 7.7 & $0.001^{*}$ \\
\hline$\%$ Energy intake total fat & 33.6 & 5.3 & 31.4 & 5.5 & $0.004^{*}$ \\
\hline$\%$ Energy intake saturated fat & 12.3 & 2.8 & 12.5 & 2.8 & $0.053^{*}$ \\
\hline \% Energy intake alcohol & 4.3 & 7.4 & 3.1 & 6.4 & $<0.0001 \dagger$ \\
\hline$\%$ Classified under-reporting energy intake & 56.0 & & 41.0 & & $<0.0001 \ddagger$ \\
\hline
\end{tabular}

*Student's t-test compared mean values between male and female participants.

†Mann-Whitney $U$ test compared median values between male and female participants.

$\mp \chi^{2}$ test compared differences between men and women for plausible and under-reporters of energy intake.

per day) (32\% vs $21 \%, \mathrm{p}<0.0001)$. For women, but not men, those classified as under-reporting EI were younger than plausible reporters: 39.2 (SD 9.4) vs 40.3 (SD 9.5) years, $\mathrm{p}=0.034$. Coders with a higher mean error rate were more likely to code food diaries classified as under-reporting EI compared with those with a lower error rate $(\mathrm{p}=0.007)$. Ethnicity, household income, diary completion, education, smoking status, length of working week, shift work and job characteristics were not significantly associated with probable underreporting for either men or women.

Stepwise logistic regression models showed that BMI, physical activity and age were significant predictors of EI under-reporting classification for men and women. Additional predictors for women were education and marital status, and weekday sitting for men. Across both sexes, BMI and physical activity were the variables that accounted for the highest increase in odds of being classified as an under-reporter. Those with a BMI of $30 \mathrm{~kg} / \mathrm{m}^{2}$ or more had higher odds of being classified as an underreporter: OR 2.66 (95\% CI 1.85 to 3.83$)$ and 5.20 (95\% CI 3.92 to 6.89 ) in women and men, respectively, compared with those with healthy BMI. Men and women in the highest physical activity category compared with the lowest were more likely to under-report: men OR 3.33 (95\% CI 2.46 to 4.50$)$; women OR 4.34 (95\% CI 2.91 to 6.55$)$ as shown in table 3 . To explore if the association between coder error rate and under-reporting was subject to potential confounding, we conducted additional logistic regression models for men and women adjusted for the variables identified in the stepwise logistic regression models. After adjustment, we did not find that a higher coder error rate was associated with increased OR of dietary intake misreporting (data not presented).

\section{Sensitivity analyses}

Two sets of sensitivity analyses (data not shown) were conducted: (1) omitting participants reporting a special diet not for weight loss and (2) omitting participants reporting a change in appetite over the last 2 weeks. Neither of the analyses modified the prevalence of potential under-reporting EI. In models excluding participants who reported being on a special diet not for weight loss, ethnic category was a significant predictor of under-reporting for women, with British Caucasians compared with other ethnic categories more likely to be classified as under-reporting EI (OR $0.5195 \%$ CI 0.27 to 0.97). In analyses removing participants reporting a change in appetite over the last 2 weeks, there was attenuation in regression estimate for weekday sitting in men, and in women, marital status was no longer a significant predictor of under-reporting. BMI and physical activity remained the strongest predictors of underreporting in all sensitivity models.

\section{DISCUSSION}

A key strength of the Airwave Health Monitoring Study is the use of 7-day food diaries to measure dietary behaviour in this large single occupation cohort. Here we provide a detailed account of the standard operating procedure developed to code the Airwave Health Monitoring Study dietary data. Dietary assessment based on self-report is inherently limited by human error (participant recall or recording, reactivity to being under surveillance), coding errors (subjective decision-making) and the limitations of nutritional databases (increasing complexity of foods consumed). Therefore, understanding sources of potential error and bias and developing a robust dietary coding methodology is essential in the generation of reliable nutritional data. We also report the prevalence of likely under-reporting of EI and its associated characteristics in a cohort of British police force employees.

\section{Dietary data generation}

To reduce intercoder error, due to the large number of coders required, and to improve coding reliability, we 
Table 2 Comparison of demographic, anthropometric, lifestyle and occupational characteristics of under-reporters and plausible reporters of energy intake across men and women in the Airwave Health Monitoring Study

\begin{tabular}{|c|c|c|c|c|c|c|c|c|c|c|}
\hline & \multicolumn{4}{|l|}{ Men } & \multirow[b]{3}{*}{ p Value* } & \multicolumn{4}{|c|}{ Women } & \multirow[b]{3}{*}{ p Value } \\
\hline & \multicolumn{2}{|c|}{$\begin{array}{l}\text { Plausible } \\
\text { reporters }\end{array}$} & \multicolumn{2}{|c|}{ Under-reporters } & & \multicolumn{2}{|c|}{$\begin{array}{l}\text { Plausible } \\
\text { reporters }\end{array}$} & \multicolumn{2}{|c|}{ Under-reporters } & \\
\hline & Mean & SD & Mean & SD & & Mean & SD & Mean & SD & \\
\hline Age at screening, years & 42.7 & 9.2 & 42.1 & 8.6 & 0.057 & 40.3 & 9.5 & 39.2 & 9.4 & 0.034 \\
\hline Basal metabolic rate (BMR), kcal day ${ }^{-1}$ & 1863 & 152 & 1940 & 157 & $<0.0001$ & 1415 & 112 & 1454 & 138 & $<0.0001$ \\
\hline \multirow[t]{2}{*}{ Daily energy intake (EI), kcal } & 2497 & 430 & 1800 & 300 & $<0.0001$ & 1942 & 314 & 1380 & 227 & $<0.0001$ \\
\hline & $\mathbf{n}$ & $\%$ & $\mathbf{n}$ & $\%$ & $\mathbf{p}^{\sim}$ & $\mathbf{n}$ & $\%$ & $\mathbf{n}$ & $\%$ & $\mathbf{p}^{\sim}$ \\
\hline Ethnicity ( $n$ 4075) & & & & & 0.338 & & & & & 0.295 \\
\hline Caucasian & 1072 & 95 & 1343 & 94 & & 861 & 97 & 589 & 95 & \\
\hline Non-Caucasian & 61 & 5 & 90 & 6 & & 31 & 3 & 28 & 5 & \\
\hline Food diary completion & & & & & 0.572 & & & & & 0.704 \\
\hline No, $<7$ days completed & 132 & 11 & 157 & 12 & & 100 & 11 & 73 & 12 & \\
\hline Yes, 7 days completed & 1001 & 89 & 1278 & 88 & & 793 & 89 & 544 & 88 & \\
\hline \multicolumn{11}{|l|}{ Body mass index } \\
\hline Healthy $\left(<25 \mathrm{~kg} / \mathrm{m}^{2}\right)$ & 342 & 30 & 217 & 15 & $<0.0001$ & 508 & 57 & 277 & 45 & $<0.0001$ \\
\hline Overweight $\left(25-30 \mathrm{~kg} / \mathrm{m}^{2}\right)$ & 634 & 56 & 809 & 56 & & 282 & 32 & 230 & 37 & \\
\hline Obese $\left(>30 \mathrm{~kg} / \mathrm{m}^{2}\right)$ & 157 & 14 & 409 & 29 & & 103 & 11 & 110 & 18 & \\
\hline Level of education ( $n$ 4077) & & & & & 0.091 & & & & & 0.058 \\
\hline Left school before taking $\mathrm{O}$ levels & 41 & 4 & 73 & 5 & & 24 & 3 & 23 & 4 & \\
\hline GCSE/O-Level/CSE' then education & 329 & 29 & 448 & 31 & & 244 & 27 & 188 & 30 & \\
\hline Vocational qualifications & 82 & 7 & 101 & 7 & & 60 & 7 & 54 & 9 & \\
\hline A levels / higher or equivalent & 359 & 32 & 464 & 32 & & 292 & 33 & 201 & 33 & \\
\hline Bachelor degree or equivalent & 255 & 23 & 264 & 18 & & 205 & 23 & 121 & 20 & \\
\hline \multirow[t]{4}{*}{ Postgraduate qualifications } & 67 & 6 & 84 & 6 & & 68 & 8 & 30 & 5 & \\
\hline & \multicolumn{4}{|c|}{ Men } & & \multicolumn{4}{|c|}{ Women } & \multirow[b]{3}{*}{ p Value $t$} \\
\hline & \multicolumn{2}{|c|}{$\begin{array}{l}\text { Plausible } \\
\text { reporters }\end{array}$} & \multicolumn{2}{|c|}{$\begin{array}{l}\text { Under- } \\
\text { reporters }\end{array}$} & & \multicolumn{2}{|c|}{$\begin{array}{l}\text { Plausible } \\
\text { reporters }\end{array}$} & \multicolumn{2}{|c|}{$\begin{array}{l}\text { Under- } \\
\text { reporters }\end{array}$} & \\
\hline & $\overline{\mathbf{n}}$ & $\%$ & $\mathbf{n}$ & $\%$ & $p$ Value $†$ & $\overline{\mathbf{n}}$ & $\%$ & $\bar{n}$ & $\%$ & \\
\hline Physical activity level (METs category) & & & & & $<0.0001$ & & & & & \multirow[t]{4}{*}{$<0.0001$} \\
\hline Low & 183 & 16 & 95 & 7 & & 164 & 18 & 51 & 8 & \\
\hline Moderate & 544 & 48 & 598 & 42 & & 462 & 52 & 282 & 46 & \\
\hline High & 406 & 36 & 742 & 51 & & 267 & 30 & 284 & 46 & \\
\hline Smoking status ( $n$ 4066) & & & & & 0.891 & & & & & 0.071 \\
\hline Never & 791 & 70 & 1004 & 70 & & 630 & 71 & 413 & 67 & \\
\hline Former & 262 & 23 & 324 & 23 & & 188 & 21 & 131 & 21 & \\
\hline Current & 76 & 7 & 102 & 7 & & 74 & 8 & 71 & 12 & \\
\hline
\end{tabular}


Table 2 Continued

\begin{tabular}{|c|c|c|c|c|c|c|c|c|c|c|}
\hline & \multicolumn{4}{|l|}{ Men } & \multirow[b]{3}{*}{$p$ Value $\dagger$} & \multicolumn{4}{|c|}{ Women } & \multirow[b]{3}{*}{$p$ Value $t$} \\
\hline & \multicolumn{2}{|c|}{$\begin{array}{l}\text { Plausible } \\
\text { reporters }\end{array}$} & \multicolumn{2}{|c|}{$\begin{array}{l}\text { Under- } \\
\text { reporters }\end{array}$} & & \multicolumn{2}{|c|}{$\begin{array}{l}\text { Plausible } \\
\text { reporters }\end{array}$} & \multicolumn{2}{|c|}{$\begin{array}{l}\text { Under- } \\
\text { reporters }\end{array}$} & \\
\hline & $\mathrm{n}$ & $\%$ & $\mathrm{n}$ & $\%$ & & $\bar{n}$ & $\%$ & $\bar{n}$ & $\%$ & \\
\hline Annual household income ( $n$ 4077) & & & & & 0.122 & & & & & 0.879 \\
\hline$<£ 32000$ & 106 & 9 & 120 & 8 & & 244 & 27 & 165 & 27 & \\
\hline$£ 32000-£ 47999$ & 140 & 12 & 152 & 11 & & 93 & 10 & 59 & 10 & \\
\hline$£ 48000-£ 57999$ & 510 & 45 & 615 & 43 & & 273 & 30 & 200 & 33 & \\
\hline$£ 58000-£ 77999$ & 265 & 23 & 388 & 27 & & 182 & 20 & 130 & 21 & \\
\hline$>£ 78000$ & 112 & 10 & 159 & 11 & & 101 & 11 & 65 & 10 & \\
\hline Marital status ( $n$ 3944) & & & & & 0.259 & & & & & 0.002 \\
\hline Cohabiting & 154 & 14 & 218 & 15 & & 150 & 18 & 151 & 25 & \\
\hline Divorced & 36 & 3 & 53 & 4 & & 78 & 9 & 35 & 6 & \\
\hline Married & 811 & 72 & 1009 & 71 & & 452 & 53 & 287 & 48 & \\
\hline Separated & 37 & 3 & 30 & 2 & & 20 & 2 & 17 & 3 & \\
\hline Single & 80 & 7 & 108 & 8 & & 161 & 18 & 106 & 18 & \\
\hline Shift work status ( $n$ 536) & & & & & 0.222 & & & & & 0.121 \\
\hline No & 57 & 34 & 78 & 42 & & 66 & 60 & 35 & 49 & \\
\hline Yes & 111 & 66 & 108 & 58 & & 45 & 40 & 36 & 51 & \\
\hline Police employment rank ( $n$ 3626) & & & & & 0.043 & & & & & 0.760 \\
\hline Police staff & 222 & 22 & 232 & 18 & & 436 & 56 & 297 & 54 & \\
\hline Police constable/sergeant & 685 & 67 & 904 & 70 & & 317 & 41 & 237 & 43 & \\
\hline Inspector/chief inspector & 93 & 9 & 126 & 10 & & 23 & 3 & 15 & 3 & \\
\hline Superintendent or above & 14 & 1 & 31 & 2 & & 3 & 0 & 1 & 0 & \\
\hline Characteristics of job role ( $n$ 2875) & & & & & 0.393 & & & & & 0.425 \\
\hline Mainly office-based duties & 458 & 42 & 337 & 40 & & 212 & 55 & 312 & 58 & \\
\hline Mainly mobile duties & 635 & 58 & 506 & 60 & & 171 & 45 & 226 & 42 & \\
\hline Working hours per week & & & & & 0.129 & & & & & 0.120 \\
\hline$<41$ hours per week & 420 & 37 & 504 & 35 & & 586 & 66 & 381 & 62 & \\
\hline 41 to 48 hour per week & 444 & 39 & 539 & 38 & & 215 & 24 & 150 & 24 & \\
\hline 49 to 54 hours per week & 138 & 12 & 187 & 13 & & 47 & 5 & 38 & 6 & \\
\hline 55 or more hours per week & 129 & 11 & 205 & 14 & & 45 & 5 & 48 & 8 & \\
\hline Quartile $(Q)$ of hours sitting per weekday & & & & & $<0.0001$ & & & & & 0.638 \\
\hline Q1 <4 hours per day & 234 & 21 & 324 & 23 & & 210 & 24 & 155 & 25 & \\
\hline Q2 $<4<6$ hours per day & 295 & 26 & 420 & 29 & & 254 & 28 & 160 & 26 & \\
\hline Q3 $\geq 6<10$ hours per day & 243 & 21 & 353 & 24 & & 229 & 26 & 154 & 25 & \\
\hline Q4 $\geq 10 \leq 13$ hours per day & 361 & 32 & 338 & 24 & & 200 & 23 & 148 & 24 & \\
\hline Coder mean error rate $(n$ 4236) $\ddagger$ & & & & & 0.007 & & & & & 0.146 \\
\hline Below average ( $<3.7 \%$ errors) & 805 & 75 & 971 & 70 & & 662 & 77 & 438 & 74 & \\
\hline Above average ( $\geq 3.7 \%$ errors) & 267 & 25 & 413 & 30 & & 193 & 23 & 153 & 26 & \\
\hline
\end{tabular}

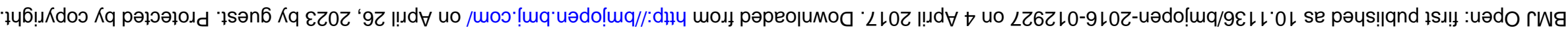


Table 3 Predictors of under-reporting energy intake by sex ${ }^{*}$ in the Airwave Health Monitoring Study

\begin{tabular}{|c|c|c|}
\hline \multicolumn{3}{|l|}{ Women (n 1286) } \\
\hline Predictor included in final model† & OR & $95 \% \mathrm{Cl}$ \\
\hline \multicolumn{3}{|l|}{ Body mass index } \\
\hline Ref: healthy $\left(<25 \mathrm{~kg} / \mathrm{m}^{2}\right)$ & 1.00 & \\
\hline Overweight $\left(25-30 \mathrm{~kg} / \mathrm{m}^{2}\right)$ & 1.74 & (1.34 to 2.27$)$ \\
\hline Obese $\left(>30 \mathrm{~kg} / \mathrm{m}^{2}\right)$ & 2.66 & (1.85 to 3.83$)$ \\
\hline \multicolumn{3}{|l|}{ Physical activity/week } \\
\hline Ref: low & 1.00 & \\
\hline Moderate & 2.06 & (1.39 to 3.06$)$ \\
\hline High & 4.34 & (2.91 to 6.55 ) \\
\hline \multicolumn{3}{|l|}{ Marital status } \\
\hline Ref: cohabiting & 1.00 & \\
\hline Divorced & 0.45 & (0.26 to 0.79$)$ \\
\hline Married & 0.63 & (0.46 to 0.87$)$ \\
\hline Separated & 0.96 & (0.45 to 2.06$)$ \\
\hline Single & 0.69 & (0.48 to 1.00$)$ \\
\hline \multicolumn{3}{|l|}{ Level of education } \\
\hline Ref: left school before taking $O$ levels & 1.00 & \\
\hline GCSE/O-level/CSE & 0.52 & $(0.25$ to 1.10$)$ \\
\hline Vocational qualifications & 0.61 & (0.27 to 1.40$)$ \\
\hline A levels/higher or equivalent & 0.40 & (0.19 to 0.84$)$ \\
\hline Bachelor degree or equivalent & 0.35 & (0.16 to 0.75$)$ \\
\hline Postgraduate qualifications & 0.25 & (0.11 to 0.60$)$ \\
\hline Age (per 5-year increase) & 0.91 & (0.84 to 0.98$)$ \\
\hline \multicolumn{3}{|l|}{ Men (n 2268) } \\
\hline \multicolumn{3}{|l|}{ Body mass index } \\
\hline Ref: healthy $\left(<25 \mathrm{~kg} / \mathrm{m}^{2}\right)$ & 1.00 & \\
\hline Overweight $\left(25-30 \mathrm{~kg} / \mathrm{m}^{2}\right)$ & 2.38 & (1.90 to 2.96$)$ \\
\hline Obese $\left(>30 \mathrm{~kg} / \mathrm{m}^{2}\right)$ & 5.20 & (3.92 to 6.89 ) \\
\hline \multicolumn{3}{|l|}{ Physical activity/week } \\
\hline Ref: low & 1.00 & \\
\hline Moderate & 2.02 & (1.50 to 2.73 ) \\
\hline High & 3.33 & (2.46 to 4.50 ) \\
\hline \multicolumn{3}{|l|}{ Quartile (Q) of hours sitting per weekday } \\
\hline Ref: Q1 < 4 hours per day & 1.00 & \\
\hline Q2 $<4<6$ hours per day & 1.10 & (0.86 to 1.41$)$ \\
\hline Q3 $\geq 6<10$ hours per day & 1.08 & (0.83 to 1.40$)$ \\
\hline$Q 4 \geq 10 \leq 13$ hours per day & 0.70 & ( 0.55 to 0.90$)$ \\
\hline Age (per 5-year increase) & 0.94 & (0.89 to 0.99 ) \\
\hline
\end{tabular}

CSE, Certificate of Secondary Education; GCSE, General Certificate of Secondary Education; O-level, ordinary level.

*Logistic regression model analyses conducted for men and women separately.

†Variables included in model presented showed significant association $(p<0.05)$ with under-reporting in stepwise logistic regression.

have developed a standard coding protocol, staff training and audit procedure. Additionally, to overcome the inherent limitations of using nutritional databases, namely that they can become outdated as food consumption becomes more varied, we have developed a standard codebook that we continually update as new foods are recorded. The mean EI and macronutrient sources of energy we have reported are comparable with those reported in the National Diet and Nutrition Survey (NDNS)..$^{28}$ The mean daily EI reported in the Airwave Health Monitoring study is 1711 (SD 395) and 2107 (SD 502) kcal for women and men, respectively, compared with 1560 (SD 442) and 2032 (SD 617) kcal for adults in the NDNS. ${ }^{28}$ Online supplementary material table S3.
Random errors may contribute to inaccurate intakes of energy and nutrients in the coding process; however, with the large sample size of the present study, we did not find coder error rate significantly associated with classification of under-reporting. The rigorous coder training, standard protocol and audit cycle have maintained a mean error rate below $10 \%$ per food diary checked. It is difficult to compare this value with other nutritional epidemiological studies as in-depth quality control results are rarely reported. Although actual error rates were not published, INTERMAP capped line errors at 6\%; however, this was based on 24-hour recall data, which cannot be directly compared with 7-day estimated weight food diaries. ${ }^{20}$ For example, during 24-hour recalls, participants can be probed to clarify intake 
information, therefore providing a more detailed record for coding.

In the present study, we did not find any association between EI misreporting and the number of food diary days completed. It has been suggested that study participants may experience 'experimenter effect' at the start of the dietary recording (days 1 and 2) and diary 'fatigue' at the end of the study period (day 7). ${ }^{29}$ Exploratory analyses (data not shown) found lower EIs on day 7 compared with day 3 of the food diary records suggesting possible 'diary fatigue' which will require further investigation. However, we did not observe that partcipants who reported a lower intake at day 7 (compared with day 3) to be more likely to be classified as under-reporting EI.

\section{Prevalence and characteristics of El misreporting}

The gold standard method to measure energy expenditure is doubly labelled water; however, this method is expensive and not feasible in large-scale population studies. By using the Goldberg method, one of the most common statistical methods for classifying implausible dietary energy reporting, we estimate that the prevalence of potential EI under-reporting to be $49 \%$ in the Airwave Health Monitoring Study cohort. As there is no valid or consensus statistical method to measure energy under-reporting in large-scale surveys published, prevalence rates vary greatly making comparison between studies problematic. The prevalence we have reported is comparable with that reported in the general British population. Applying individualised PAL values and calculating 95\% CI to estimate acceptability of EI reporting Murakami et $a \vec{l}^{0}$ classified $45 \%$ men and $55 \%$ women as under-reporting EI from 7-day weighed food diaries. However, these rates are greater than those published in a review of EI misreporting that found the prevalence of under-reporting to be between 12 and $44 \%$ in studies using estimated food records conducted for 3, 4 or 7 days. ${ }^{31}$ Studies included in the review applied estimated PAL values of between 1.05 and 1.35. The PAL applied can be arbitrary in the absence of objective PAL measurement information; because of the way that the Goldberg equation derives the under-reporting cut-off points (assuming energy balance), the PAL used will impact the prevalence of under-reporting. Owing to the likely heterogeneous job roles within the Airwave Health Monitoring Study cohort (eg, office based, on the beat officers and mobile patrol), we decided to use the MET data to estimate PAL levels rather than apply a universal value. The higher prevalence of under-reporting that we have observed in comparison with some previous studies is likely due to the considerably higher PAL values (1.4, 1.6 and 1.8) we have applied to the Airwave Health Monitoring Study Cohort. A PAL of 1.4 was selected as the lowest value as it is representative of a man or a woman with a sedentary non-occupational activity level and light occupational level of activity Therefore, the positive association between higher PAL and possible under-reporting that we observed may be due to the selfreported measure of physical activity used.

A systematic review found that self-report physical activity measurements have low to moderate correlations with direct measurements with under-reporting and over-reporting of physical activity observed and no bias towards a specific population group. ${ }^{32}$ In the current study, we applied identical MET values for each activity level recorded to all classes of BMI based on IPAQ-SF guidelines (3.3 walking, 4.0 moderate-intensity activity and 8.0 vigorous-intensity activity). A recent study has suggested that calculating METs using the standard resting oxygen uptake of $3.5 \mathrm{~mL} \mathrm{O}_{2}^{-1} \mathrm{~min}^{-1}$ could overestimate energy expenditure in overweight and obese people by up to $38.8 \%,{ }^{33}$ consequently overestimating under-reporting in these individuals. Systematic overestimation of METs in obese participants may result in misclassification of PAL category, subsequently overestimating and biasing classification of underreporting in obese participants.

In agreement with previous studies, we observed that classification of under-reporting EI was biased towards specific population groups. Consistent with European Prospective Investigation into Cancer (EPIC)-Norfolk ${ }^{34}$ and UK NDNS, ${ }^{9}$ we found under-reporting prevalence to be directly associated with BMI. In agreement with a previous study conducted in a working-age French cohort, higher education in women, but not men, was associated with plausible EI reporting. ${ }^{35}$ We found advancing age to be a weak but significant predictor of plausible EI reporting, in agreement with findings from the EPIC cohort, ${ }^{11}$ although other studies have shown advancing age to be associated with under-reporting. ${ }^{10}$ The Whitehall II study reported that those in lower employment grades were more likely to be classified as reporting low EIs, following adjustment for BMI. ${ }^{36}$ However, in our logistic regression models, we did not find the occupational variables of rank, or job role to be significantly associated with classification of underreporting EI after adjustment for established confounders. Higher mean weekday sitting hours for men was associated with reduced odds of being classified as under-reporting. Participants in the highest quartile for weekday siting were more likely to be in a job role that is predominantly office based potentially making it more practical for the participant to record dietary intake compared with being on mobile duties; however, in the current sample, $\sim 30 \%$ of participants did not have these data available potentially explaining why job role was not found to be a significant predictor of EI reporting.

To avoid the potential excessive exclusion of participants based on EI misreporting, a previous study compared a simplistic measure of dietary reporting plausibility with the Goldberg method. ${ }^{37}$ They reported that arbitrary cut-off points set at $<500 \mathrm{kcal} /$ day for under-reporting and $>3500 \mathrm{kcal} /$ day for over-reporting classified $1 \%$ as misreporters compared with $31 \%$ using the Goldberg equation. ${ }^{37}$ Moreover, excluding 
misreporters based on the Goldberg method, compared with the simple cut-off method did not substantially alter the relationship between recorded dietary intakes with biomarkers of intake. ${ }^{37}$ In the present study, we excluded two participants that had a mean intake of $<500 \mathrm{kcal} /$ day. There were $32(0.7 \%)$ participants that reported a mean EI of $>3500 \mathrm{kcal}$ per day. Based on the Goldberg equation, these 32 participants were classified as reliable reporters of EI, potentially due to the majority of these participants reporting moderate and high PAL.

\section{Future work}

Although our results reinforce the suggestion that potential under-reporting of EI is not a result of random error but a systematic bias, it is important to note that our results may reflect in part, the result of the analytical procedure used to classify EI misreporting. ${ }^{38}$ In particular, the association between PAL and BMI with under-reporting are potentially subject to statistical artefact. An important feature of equations that use estimated energy expenditure to determine plausibility of energy reporting is that they need to be reflective of the population to which they are applied. In the absence of inexpensive and convenient biomarkers to determine EI, there is a need to investigate appropriate algorithms to determine BMR and energy expenditure of representative populations.

The present study only considers EI misreporting; however, under-reporting may not be distributed equally across all types of foods but may be biased towards 'unhealthy' foods. ${ }^{39}$

A large pooling study of cohort studies found that BMI was a strong predictor of protein and EI underreporting against established biomarkers (24-hour urinary nitrogen and doubly labelled water) ${ }^{40}$ However, in the absence of effective biomarkers to use in large cohort studies, bias in reporting at the food level in freeliving populations cannot be estimated using statistical methods, or therefore adjusted for in analyses. The collection of biological samples (spot urine and blood) as part of the Airwave Health Monitoring Study will allow for future exploratory investigations into the use of biomarkers to aid the assessment of dietary intake in large epidemiological studies.

\section{Study limitations}

There are a number of limitations specific to the current study. First, previous research has shown that those classified as restrained eaters are more likely to under-report their EI. ${ }^{41}$ A study investigating restrained eating in the UK NDNS reported that men in nonmanual occupations were more likely to be classified as having a higher restrained eating score, and that in men, but not women, this trait was associated with under-reporting. ${ }^{42}$ Additionally, it has been suggested that stress may play a role in restrained eating behaviours. ${ }^{43}$ Therefore, as specific job roles within the police force may be associated with higher levels of stress, this could be an important consideration.
However, we did not observe any difference in underreporters, plausible reporters and job role. Although questions about dietary restraint ${ }^{44}$ were not included as part of the health screen, sensitivity analyses were conducted excluding those following a special diet not for weight loss or a change in appetite during the previous 2 weeks. These analyses did not change the overall prevalence of under-reporting of EI but did alter the significance of predictive characteristics. However, in both sets of sensitivity analysis, BMI and PAL remained the strongest predictors of under-reporting.

A further limitation of our current study is missing data on specific variables, in particular shift work that is only currently available for $\sim 12 \%$ of participants. However, shift work is highly associated with job role and rank within the cohort, neither of which were found to be a predictor of energy misreporting. Finally, it could be argued that the dietary data generated from the Airwave Health Monitoring Study cohort lacks external validity as it is based on a specific occupational group. However, with 250000 people in Great Britain being employed by the police force in $2012,{ }^{45}$ and with recent interest in reducing obesity in public sector workers, ${ }^{46}$ the data produced will give a valuable insight into dietary habits and potential measurement bias in comparable occupational groups such as paramedics and fire fighters.

\section{CONCLUSION}

The Airwave Health Monitoring Study has collected the largest single occupation prospective dietary data set in the UK. Despite the acknowledged limitations of selfreported dietary intake, it is currently the only method of dietary measurement that is feasible for deployment in large-scale nutritional epidemiological studies. Here we provide a detailed account of the standard operating procedure developed to code the dietary data in the Airwave Health Monitoring Study, which we believe gives a useful insight into the practicalities of reducing coding error in large-scale dietary studies using food records. In agreement with previous studies, we observed the prevalence of under-reporting of EI to be directly associated with BMI. The reasons for this association are likely to be multifactorial and related to participant and methodological factors. However, with the potential for bias due to under-reporting it would be prudent to conduct sensitivity analyses ${ }^{31}$ or to adjust for EI ${ }^{47}$ in analyses of dietary factors in relation to disease outcomes. The availability of blood and urine samples in the Airwave Health Monitoring Study, together with the dietary data, provides a valuable resource to investigate nutritional biomarkers for use in future studies.

\section{Author affiliations}

${ }^{1}$ Nutrition and Dietetic Research Group, Faculty of Medicine, Imperial College, London, UK

${ }^{2}$ Department of Epidemiology and Biostatistics, MRC-PHE Centre for Environment and Health, School of Public Health, Imperial College, London, UK 
Acknowledgements We thank all participants in the Airwave Health Monitoring Study. We would also like to thank all the dietary coders who have contributed to the generation of the dietary data: Shatha Alrabiah, Jessica Ayling, Andrea Carames, Zhengyu Fan, Kirsty Frost, Louise Hirichi, Zanna Hofstede, Niamh O'Sullivan, Kristina Petersen, Claudia Schreuder, Manny Singh, Elizabeth Slack, Jill Twomey, Alan Wan, Jessica Ware and Yiling Zhu. We also thank Andy Heard, database manager, Deepa Singh, clinical lead who set up the clinics and Louisa Cavaliero who assisted in data collection and management.

Contributors GF, RG, QC and RE formulated the research question and methodological design; $R G$. was responsible for data analysis and drafting of the manuscript. GF, PE and A-CV contributed to the interpretation of results and final manuscript. RG, RE, YM and $K L$ contributed to the development of the food diary coding protocol, coder training and audit checking. QC assisted RE and RG with the dietary data extraction and cleaning. MA and JS were responsible for the validation of the non-dietary data extracts used in the analyses. PE is the principal investigator of the Airwave Health Monitoring Study. All authors read and approved the final manuscript.

Funding The Airwave Health Monitoring Study is funded by the Home Office (grant number 780- TETRA) with additional support from the National Institute for Health Research (NIHR). The diet coding was supported through discretionary departmental funds. This paper presents independent research funded by the Home Office with additional support from the NIHR, ICHNT and Imperial College Biomedical Research Centre (BRC) at Imperial College Healthcare NHS Trust. P.E. and G.F. are NIHR senior investigators. P.E. acknowledges support from the NIHR Biomedical Research Centre at Imperial College Healthcare NHS Trust and Imperial College London, the NIHR Health Protection Research Unit on Health Effects of Environmental Hazards and the Medical Research Council-Public Health England (MRC-PHE) Centre for Environment and Health.

This work used computing resources of the UK MEDical BIOinformatics partnership-aggregation, integration, visualisation and analysis of large, complex data (UK MED-BIO) which is supported by the Medical Research Council (grant number MR/L01632X/1). The Section of Endocrinology and Investigative Medicine is funded by grants from the MRC, Biotechnology and Biological Sciences Research Council (BBSRC), NIHR, an Integrative Mammalian Biology (IMB) Capacity Building Award, an FP7-HEALTH-2009-241592 EuroCHIP grant and is supported by the NIHR Biomedical Research Centre Funding Scheme.

Disclaimer The views expressed are those of the authors and not necessarily those of the Home Office, ICHNT, the BRC, the NHS, the NIHR or the Department of Health.

Competing interests None declared.

Ethics approval National Health Service Multi-site Research Ethics Committee (MREC/13/NW/0588).

Provenance and peer review Not commissioned; externally peer reviewed.

Data sharing statement No additional data are available.

Open Access This is an Open Access article distributed in accordance with the terms of the Creative Commons Attribution (CC BY 4.0) license, which permits others to distribute, remix, adapt and build upon this work, for commercial use, provided the original work is properly cited. See: http:// creativecommons.org/licenses/by/4.0/

\section{REFERENCES}

1. Elliott $P$, Vergnaud AC, Singh D, et al. The Airwave Health Monitoring Study of police officers and staff in Great Britain: rationale, design and methods. Environ Res 2014;134:280-5.

2. Cohort Strategic Review Subgroup. Maximising the value of UK population cohorts. MRC strategic review of the largest UK population cohort studies. Swindon: Medical Research Council, 2014.

3. Fung TT, McCullough M, van Dam RM, et al. A prospective study of overall diet quality and risk of type 2 diabetes in women. Diabetes Care 2007;30:1753-7.
4. Rimm EB, Katan MB, Ascherio A, et al. Relation between intake of flavonoids and risk for coronary heart disease in male health professionals. Ann Intern Med 1996;125:384-9.

5. Aljuraiban GS, Chan Q, Oude Griep LM, et al. The impact of eating frequency and time of intake on nutrient quality and body mass index: the INTERMAP study, a population-based study. J Acad Nutr Diet 2015;115:528-36.

6. Kim S, Park GH, Yang JH, et al. Eating frequency is inversely associated with blood pressure and hypertension in Korean adults: analysis of the Third Korean National Health and Nutrition Examination Survey. Eur J Clin Nutr 2014;68:481-9.

7. Mekary RA, Giovannucci E, Cahill L, et al. Eating patterns and type 2 diabetes risk in older women: breakfast consumption and eating frequency. Am J Clin Nutr 2013;98:436-43.

8. Pot GK, Hardy R, Stephen AM. Irregular consumption of energy intake in meals is associated with a higher cardio-metabolic risk in adults of a British birth cohort. Int J Obes 2014;38:1518-24.

9. Rennie KL, Coward A, Jebb SA. Estimating under-reporting of energy intake in dietary surveys using an individualised method. Br J Nutr 2007;97:1169-76.

10. Briefel RR, Sempos CT, McDowell MA, et al. Dietary methods research in the third National Health and Nutrition Examination Survey: underreporting of energy intake. Am J Clin Nutr 1997;65 (Suppl 4):1203S-9S.

11. Ferrari P, Slimani N, Ciampi A, et al. Evaluation of under- and overreporting of energy intake in the 24-hour diet recalls in the European Prospective Investigation into Cancer and Nutrition (EPIC). Public Health Nutr 2002;5:1329-45.

12. Rosell MS, Hellénius ML, de Faire UH, et al. Associations between diet and the metabolic syndrome vary with the validity of dietary intake data. Am J Clin Nutr 2003;78:84-90.

13. Braakhuis AJ, Meredith K, Cox GR, et al. Variability in estimation of self-reported dietary intake data from elite athletes resulting from coding by different sports dietitians. Int J Sport Nutr Exerc Metab 2003;13:152-65.

14. Lachat C, Hawwash D, Ocké MC, et al. Strengthening the Reporting of Observational Studies in Epidemiology-Nutritional Epidemiology (STROBE-nut): An Extension of the STROBE Statement. PLoS Med 2016;13:e1002036.

15. Bingham SA, Gill C, Welch A, et al. Validation of dietary assessment methods in the UK arm of EPIC using weighed records, and 24-hour urinary nitrogen and potassium and serum vitamin $\mathrm{C}$ and carotenoids as biomarkers. Int J Epidemiol 1997;26(Suppl 1): S137-51.

16. Nelson M, Haraldsdottir J. Food photographs: practical guidelines 11: development and use of photographic atlases for assessing food portion size. Pub Health Nutr 1998;1:231-7.

17. Food Standards Agency. Foodbase. 2005. http://www.foodbase.org. uk/results.php?f_report_id=82 (accessed November 2013).

18. Food Standards Agency. Food portion sizes. 3rd edn. London: TSO, 2010.

19. Charrondiere R, Haytowitz D, Stadlmayr B. FAO/INFOODS density database Version 2.0. 2012. http://www.fao.org/docrep/017/ap815e/ ap815e.pdf (accessed November 2013).

20. Conway R, Robertson C, Dennis B, et al. Standardised coding of diet records: experiences from INTERMAP UK. Br J Nutr 2004;91:765-71.

21. Australian Bureau of Statistics. Australian Health Survey: Users' guide. 2013. http://www.abs.gov.au/ausstats/abs@.nst/Lookup/4363. 0.55.001Chapter65142011-13 (accessed August 2015).

22. Kivimäki M, Jokela M, Nyberg ST, et al. Long working hours and risk of coronary heart disease and stroke: a systematic review and meta-analysis of published and unpublished data for 603838 individuals. Lancet 2015;386:1739-46.

23. World Health Organisation Obesity: preventing and managing the global epidemic. Report of a WHO consultation on obesity, Geneva, 3-5 June 1997. Geneva: WHO, 2008.

24. Craig CL, Marshall AL, Sjöström M, et al. International physical activity questionnaire: 12-country reliability and validity. Med Sci Sports Exerc 2003;35:1381-95.

25. International Physical Activity Questionnaire scoring protocol. 2005. http://www.ipaq.ki.se/scoring.htm (accessed October 2014)

26. Committee on Medical Aspects of Food Policy. Dietary Reference Values for Food Energy and Nutrients for the United Kingdom: Report of the Panel on Dietary Reference Values of the Committee on Medical Aspects of Food Policy. London: H.M. Stationery Office, 1991.

27. Black AE. Critical evaluation of energy intake using the Goldberg cut-off for energy intake:basal metabolic rate. A practical guide to its calculation, use and limitations. Int J Obes Relat Metab Disord 2000;24:1119-30. 
28. Bates B, Lennox A, Prentice A, et al. National Diet and Nutrition Survey: results from years 1-4 (combined) of the Rolling Programme (2008/2009-2011/12). London: TSO, 2014.

29. Biltoft-Jensen A, Matthiessen J, Rasmussen LB, et al. Validation of the Danish 7-day pre-coded food diary among adults: energy intake $\mathrm{v}$ energy expenditure and recording length. Br J Nut 2009;102:1838-46.

30. Murakami K, McCaffrey TA, Livingstone MBE. Associations of dietary glycaemic index and glycaemic load with food and nutrient intake and general and central obesity in British adults. Br J Nutr 2013;110:2047-57

31. Poslusna K, Ruprich J, de Vries JHM, et al. Misreporting of energy and micronutrient intake estimated by food records and 24 hour recalls, control and adjustment methods in practice. Br J Nutr 2009;101(Suppl 2):S73-85.

32. Prince SA, Adamo KB, Hamel ME, et al. A comparison of direct versus self-report measures for assessing physical activity in adults: a systematic review. Int J Behav Nutr Phys Act 2008;5:56

33. Wilms B, Ernst B, Thurnheer M, et al. Correction factors for the calculation of metabolic equivalents (MET) in overweight to extremely obese subjects. Int J Obes 2014;38:1383-7.

34. Lentjes $\mathrm{MAH}, \mathrm{McT}$ Taggart $\mathrm{A}$, Mulligan AA, et al. Dietary intake measurement using $7 \mathrm{~d}$ diet diaries in British men and women in the European Prospective Investigation into Cancer-Norfolk study: a focus on methodological issues. $\mathrm{Br} \mathrm{J}$ Nutr 2014;111:516-26.

35. Berta Vanrullen I, Volatier JL, Bertaut A, et al. Characteristics of energy intake under-reporting in French adults. Br J Nutr 2014;111:1292-302.

36. Stallone DD, Brunner EJ, Bingham SA, et al. Dietary assessment in Whitehall II: the influence of reporting bias on apparent socioeconomic variation in nutrient intakes. Eur J Clin Nutr 1997;51:815-25.
37. Rhee JJ, Sampson L, Cho E, et al. Comparison of methods to account for implausible reporting of energy intake in epidemiologic studies. Am J Epidemiol 2015;181:225-33.

38. Murakami K, Livingstone MBE. Prevalence and characteristics of misreporting of energy intake in US adults: NHANES 2003-2012. Br J Nutr 2015;114:1294-303.

39. Lafay L, Mennen L, Basdevant A, et al. Does energy intake underreporting involve all kinds of food or only specific food items? Results from the Fleurbaix Laventie Ville Santé (FLVS) study. Int J Obes Relat Metab Disord 2000;24:1500-6.

40. Freedman LS, Commins JM, Moler JE, et al. Pooled results from 5 validation studies of dietary self-report instruments using recovery biomarkers for energy and protein intake. Am J Epidemiol 2014;180:172-88

41. McCrory MA, Hajduk CL, Roberts SB. Procedures for screening out inaccurate reports of dietary energy intake. Public Health Nutr 2002;5:873-82.

42. Lopez ALO, Johnson L. Associations between restrained eating and the size and frequency of overall intake, meal, snack and drink occasions in the UK adult national diet and nutrition survey. PLOS ONE 2016;11:e0156320.

43. Yau YHC, Potenza MN. Stress and Eating Behaviours. Minerva Endocrinol 2013;38:225-67.

44. Stunkard AJ, Messick S. The three-factor eating questionnaire to measure dietary restraint, disinhibition and hunger. J Psychosom Res 1985;29:71-83.

45. Association of Chief Police Officers. UK Police Directory 2012. Chichester, UK: Keyways Publishing Ltd, 2012.

46. Stevens S. Get serious about obesity or bankrupt the NHS. 2014. http://www.england.nhs.uk/2014/09/17/serious-about-obesity (accessed October 2014).

47. Willett W, Howe G, Kushi L. Adjustment for total energy intake in epidemiologic studies. Am J Clin Nutr 1997;65:1220S-8S; discussion 1229S-1231S. 